\title{
PHOTOIONIZED PLASMAS IN X-RAY BINARY PULSARS:
}

\section{ASCA OBSERVATIONS}

FUMIAKI NAGASE

The Institute of Space and Astronautical Science, 3-1-1 Yoshinodai, Sagamihara, Kanagawa 229, Japan

\section{Introduction}

Massive X-ray binary pulsars have often evolved early-type companion stars which emanate strong stellar winds. X-rays emitted from the accreting neutron star irradiate and ionize the surrounding stellar wind, thus forming a photoionized sphere surrounding the neutron star. The photoionization structure of matter surrounding the neutron star was calculated by Hatchett and McCray (1977) and McCray et al. (1984), for Cen X-3 and Vela $\mathrm{X}-1$ respectively.

Emission lines from He-like and hydrogenic ions are expected to be emitted from such plasmas due to radiative recombination followed by cascades. The spectroscopy of emission lines in X-ray binary systems is useful for investigating the ionization structure of the photoionized region surrounding the neutron star.

Before the $A S C A$ observations, however, only an iron $\mathrm{K} \alpha$ emission line at $6.4 \mathrm{keV}$ was observed for several X-ray pulsars (e.g., Nagase 1989). Using data obtained with the SIS sensors (CCD cameras) onboard $A S C A$, emission lines from various elements, such as $\mathrm{Mg}, \mathrm{Si}, \mathrm{S}, \mathrm{Ar}, \mathrm{Ca}$, and $\mathrm{Fe}$ are detectable and even $\mathrm{K} \alpha$ lines from ions of different ionization states become resolvable owing to the high sensitivity and energy resolution of the CCD cameras.

\section{Emission Lines from Photoionized Plasma}

A prominent fluorescent $\mathrm{K} \alpha$ line was detected at $6.40 \mathrm{keV}$ in the spectrum of GX $1+4$, yielding the estimate for the ionization degree of iron to be lower than Fe IV (Kotani et al 1997). From the SIS spectrum of GX 301-2 
it was found that this source exhibits fluorescent $\mathrm{K} \alpha$ lines of $\mathrm{Si}, \mathrm{S}, \mathrm{Ar}$, and $\mathrm{Ca}$ as well the $\mathrm{K} \alpha$ line of iron (Saraswat et al. 1996). This result yields an estimate for the ionization parameter of the effective emission site to be $\log \xi \approx 1.5$, thus suggesting a cold matter for reprocessing.

In contrast to these results, an interesting feature of the emission lines was observed in the spectrum of Vela X-1, which was observed during the eclipse phase (Nagase et al. 1994). The spectrum unfolded with the best-fit model is shown in Figure 1.

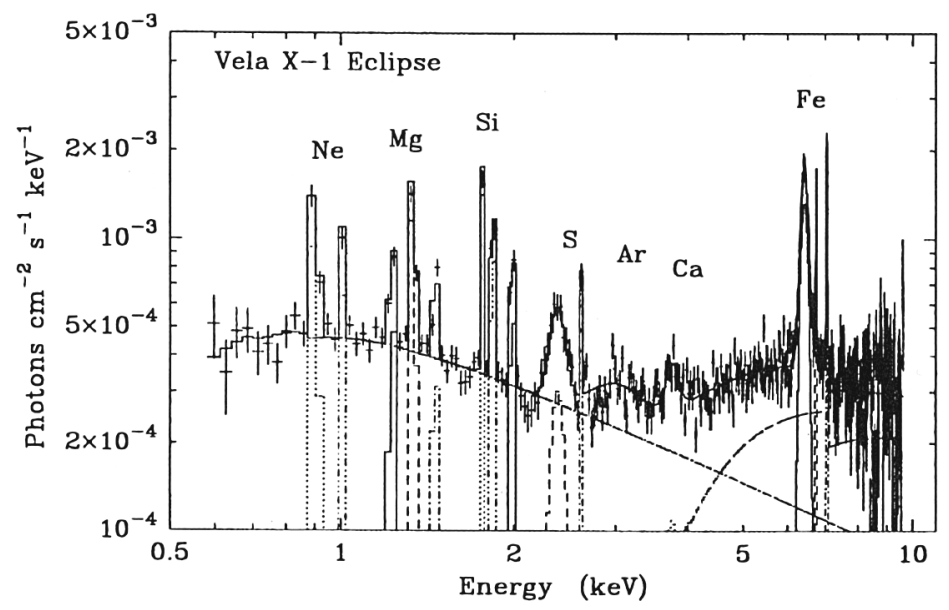

Figure 1. The ASCA unfolded SIS spectrum of Vela X-1 obtained during eclipse phase. The model consists of two component continua and fifteen lines.

From this spectrum we can resolve 15 lines superposed on the flat continuum which can be modeled by two (with high and low absorption column densities) power laws. $\mathrm{K} \alpha$ lines of $\mathrm{Ne}, \mathrm{Mg}, \mathrm{Si}, \mathrm{S}, \mathrm{Ar}, \mathrm{Ca}$, and $\mathrm{Fe}$ are detected and mostly dominated by the recombination lines of He-like ions for these elements, except for the iron for which the $6.4-\mathrm{keV}$ fluorescent $\mathrm{K} \alpha$ line by cold matter is dominant. This result suggests that radiative recombination followed by cascades is the dominant process in the X-ray irradiated stellar wind, since such a stellar wind forms highly ionized zones of He-like and hydrogenic ions with a relatively low electron temperature of about $100 \mathrm{eV}$ (Kallman \& McCray 1982). From the present result, the photoionization parameter of the effective emission site is estimated to be $\log \xi \approx 2.3$, which is consistent with the calculation by McCray et al. (1984).

From the SIS spectrum of Cen X-3 obtained with $A S C A$, three iron lines are clearly resolved at $6.4 \mathrm{keV}, 6.7 \mathrm{keV}$, and $6.96 \mathrm{keV}$, identifying the fluorescent $\mathrm{K} \alpha$ line of cold matter, and the recombination $\mathrm{K} \alpha$ lines of He-like and hydrogenic ions of iron, respectively. Interestingly, the relative intensities of the three lines change along the orbital phase. In addition 
to the iron lines, lines of $\mathrm{Ne}, \mathrm{Mg}, \mathrm{Si}$, and $\mathrm{S}$ are also observed at lower energies and these are identified to the recombination $\mathrm{K} \alpha$ lines of hydrogenic ions. From the $A S C A$ spectrum, the ionization parameter of the effective emission site is estimated as $\log \xi \approx 3.4$ for Cen X-3 (see Ebisawa et al. 1996 for details). Hence, the degree of photoionization of Cen X-3 is much higher than that of Vela X-1. The $\xi$-value derived is consistent with the calculation by Hatchett and McCray (1977).

The different emission line features typically seen in the three HMXB pulsars, GX301-2, Vela X-1, and Cen X-3 can be understood by the difference of the binary size and the X-ray luminosity of the source (see e.g., Nagase 1997).

\section{Recombination Continuum}

In the above consideration of Vela X-1 spectrum, we did not mention the emission due to the transition of free electrons directly to the ground level of the He-like or hydrogenic ions. The probabilities of these free-bound transitions are not negligible compared with the cascade recombination probabilities. Since the electron temperature of the photoionized stellar wind is as low as $\sim 100 \mathrm{eV}$, such free-bound transitions will produce broad emission-line-like features at the recombination edges.

The broad features at the $\mathrm{S}$ and Ar bands $(2.4$ and $3.0 \mathrm{keV})$ in the Vela $\mathrm{X}-1$ spectrum (see Fig. 1) are the evidence of the free-bound emission of $\mathrm{He} / \mathrm{H}$-like ions of $\mathrm{Si}$ and $\mathrm{S}$. A broad emission line feature observed at 1.42 $\mathrm{keV}$ in the $4 \mathrm{U} 1626-67$ spectrum is consistent with the free-bound emission of hydrogenic neon (Angelini et al. 1995). Better and clearer evidence of the recombination continuum emission that mimics a line feature was obtained from the Cyg X-3 spectrum (Liedahl and Paerels 1996; Kawashima and Kitamoto 1996). From these broad line features the plasmas surrounding these system are confirmed to be in highly photoionized states due to X-ray irradiation.

\section{4U1538-52 and OAO1657-415}

4U1538-52 and OAO1657-415 are eclipsing X-ray binary pulsars with binary parameters and X-ray lunminosities similar to those of Vela X-1. $A S C A$ observed the two pulsars during their eclipses with the hope that they would show recombination-lines dominated spectra similar to that of Vela X-1.

As seen in Figure 2, the observed spectra are both dramatically different from each other and from the Vela X-1 spectrum (Fig. 1) against expectation. Excess of soft X-ray continuum is seen in the 4 U1538-52 eclipse spectrum. This is due to contamination by interstellar dust scattering. No 
conspicuous line was detected at $\mathrm{Mg}, \mathrm{Si}$ or $\mathrm{S}$. Only an intense $6.4-\mathrm{keV}$ fluorescent line of iron was detected and He-like and hydrogenic Ne lines are marginally seen (see Nagase et al. 1997 for details).
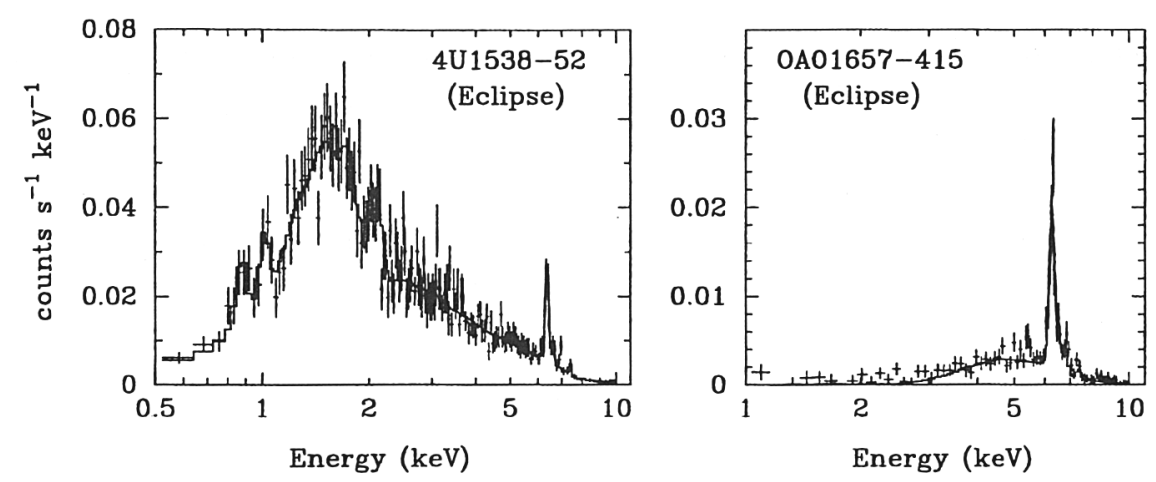

Figure 2. The ASCA SIS spectra of 4U1538-52 and OAO1657-415 obtained during their eclipse phases. The solid lines represents the best fit model to the data.

The spectrum of OAO1657-415 observed during eclipse shows a further drastically different feature. It exhibits only an intense iron line at $6.4 \mathrm{keV}$. Even the scattering continuum is weak and highly absorbed at energies below $3 \mathrm{keV}$. No evidence of recombination lines of $\mathrm{Mg}, \mathrm{Si}$, and $\mathrm{S}$ is seen.

These differences in eclipse spectra may be due to the delicate balance of their circumstellar environments, such as the wind density, the X-ray luminosity and chemical abundance of elements. Present results suggest that the $A S C A$ spectroscopy is extremely suitable to investigate the ionization structure of matter surrounding the neutron star in wind-fed pulsars.

\section{References}

Angelini, L., et al. (1995) Astrophys. J. 449, L41

Ebisawa, K., et al. (1996) Publ. Astron. Soc. Japan 48425

Hatchett, S. \& McCray, R. (1977) Astrophys. J. 211, 552

Kawashima, K., \& Kitamoto, S. (1996) Publ. Astron. Soc. Japan 48, L113

Kotani, T., et al. (1997) Astrophys. J. submitted

Kallman, T.R., \& McCray, R. (1982) Astrophys. J. Suppl. 50, 263

Liedahl, D.A., \& Paerels, F. (1996) Astrophys. J. 468, L33

McCray, R., Kallman, T.R., Castor, TJ.I., \& Olsen, G.L. (1984) Astrophys. J. 282, 245 Nagase, F., (1989) Publ. Astron. Soc. Japan 36, 1

Nagase, F., (1997) in X-Ray Imaging and Spectroscopy of Cosmic Hot Plasmas, Proc. of the Internat. Symp. on X-ray Astronomy (March 11-14, 1996, Waseda Uni. Tokyo), eds., F. Makino and K. Mitsuda (Universal Academy press, Inc.), p. 419

Nagase, F., et al. (1994) Astrophys. J. 436, L1

Nagase, F., et al. (1997) in preparation

Saraswat, P., et al. (1996) Astrophys. J. 463, 726 